\title{
Prostatic carcinomas with neuroendocrine differentiation diagnosed in needle biopsies, a morphologic study of 7 cases among 465 sequential biopsies in a tertiary cancer center
}

\author{
MVA Lima, C Nogueira, JAA Oliveira, FJ Muniz Neto, M Franco, F Tavora \\ Hospital do Câncer, Instituto do Câncer do Ceara (MVAL, CN, JAAO, FJMN, FT), Fortaleza, Cea- \\ ra and Escola Paulista de Medicina (MF, FT), São Paulo, Brazil
}

\begin{abstract}
Purpose: Neuroendocrine carcinomas (NEC) of the prostate are rare, with only a few series hitherto reported. The objective of this study was to assess in a single institution the clinical and morphologic characteristics of neuroendocrine carcinomas diagnosed in needle core biopsies.

Materials and Methods: The current study analyses seven cases diagnosed in needle biopsies at a large tertiary regional cancer center from Northeastern Brazil. Two pathologists reviewed specimens retrospectively, and demographic and morphologic characteristics were compared to 458 acinar tumors diagnosed in the same period.

Results: There were five small cell carcinomas and two low-grade neuroendocrine carcinomas (carcinoid). NEC were associated with an acinar component in 5/7 cases and the Gleason score of the acinar component was always $>6$. The number of cores involved in prostates with NEC was greater $(65 \%$ compared to $24 \%$ of acinar tumors, $p<0.05)$. The mean PSA at diagnosis was 417.7 (range 5.7-1593, SD 218.3), compared to $100.5(\mathrm{p}=0.1$ ) of acinar tumors (range 0.38545, SD 22.7). Prostates harboring NEC were bigger ( $<<0.001$, mean volume $240 \mathrm{~mL}$ vs. $53 \mathrm{~mL}$ of acinar tumors). Treatment of NEC included palliative surgery, chemotherapy, and hormonal therapy.

Conclusions: NEC of the prostate is rare and often associated with a high-grade acinar component. Prostates with NEC tend to be larger and involve a greater number of cores than acinar tumors. PSA at diagnosis does not seem to predict the presence of NE tumors in needle biopsy.
\end{abstract}

Key words: prostate; neoplasms; carcinoid tumor; neuroendocrine tumor; treatment outcome Int Braz J Urol. 2011; 37: 598-604

\section{INTRODUCTION}

Neuroendocrine carcinomas (NEC) of the prostate are rare, representing less than $0.5 \%$ of prostate carcinomas in the few series reported to date (1-8). The current classification of neuroendocrine carcinomas is based on the World Health Organization 2004 lung tumor classification, and it divides those tumors into well-differentiated neuroendocrine carcinomas (carcinoid tumors), moderately-differentiated neuroendocrine carcinomas (atypical carcinoid tumors), and poorly-differentiated neuroendocrine carcinomas, which include two morphologic distinct entities (small cell carcinomas and large cell neuroendocrine carcinomas) (9). The classification is based solely on histomorphology and relies on both light microscopy and immunohistochemical studies.

Albeit rare, the most common neuroendocrine carcinoma of the prostate is by far small cell carcinoma. Furthermore, it is estimated that up to $10 \%$ of prostate cancer in patients with androgen-resistant disease after long-term androgen deprivation therapy are high grade NEC, most with associated acinar adenocarcinoma (10).

Recognition of this entity via needle biopsies is critical, as its therapy differs significantly from that of usual acinar high-grade prostatic adenocarcinoma. 


\section{MATERIALS AND METHODS}

Seven cases of neuroendocrine carcinomas primary to the prostate were collected over 4 years (2006-2010) from the Department of Pathology archives of the Cancer Hospital of the Ceara Cancer Institute among 465 sequential needle biopsies from the in-house Urology Department Service. Patients with neuroendocrine tumors primary to other sites were excluded from the study.

The morphologic data independently collected and reviewed by two pathologists (FT and CDM) were basic morphology to include small or non-small cell pattern, percentage of cores involved, association with conventional acinar adenocarcinoma and the Gleason grading, mitotic rate, presence of tumor necrosis and any other morphologic findings.

Immunohistochemical studies were performed on the available paraffin blocks in all seven cases. Immunohistochemistry was performed in our laboratory using the standard streptavidin-biotinperoxidase procedure. Primary monoclonal antibodies to PSA (dilution 1:100), chromogranin (1:2000), synaptophysin (1:50), ki67 (1:100) and p63 (1:300) (Dako Inc., Carpinteria, USA) were applied to 5-mm thick $10 \%$ formalin-fixed, paraffin-embedded tissue sections. The sections underwent a process of deparaffinization, rehydration, and washing in xylene, graded alcohols, and distilled water. Blockage of endogenous peroxide activity was performed by incubation with $3 \% \mathrm{H}_{2} \mathrm{O}_{2}$. The sections were placed in 10 $\mathrm{mM}$ citrate buffer at $\mathrm{pH} 6$ with subsequent antigen retrieval procedure. The antigen-antibody reaction was visualized using the avidin-biotin peroxidase complex and diaminobenzidine as the chromogen. Slides were counterstained with hematoxylin. Positive results consisted of dark brown nuclear (p63, ki67) and cytoplasmic (chromogranin, synaptophysin) staining and cytoplasmic and luminal granular staining of secretory epithelial cells by PSA. Appropriate positive and negative controls were included. Only staining that was moderate or strong was considered positive.

Clinical follow-up was possible in all but one case by retrospective clinical chart review by one of the authors (MVL).

Clinical and histopathologic variables were compared among categorized groups using the $\chi^{2}$ test or Student t- test. A p value less than 0.05 was considered significant. The software SPSS 5.0 (Chicago, IL) was used for statistical analyses.

\section{RESULTS}

The clinical and pathological characteristics of the seven cases are summarized in Tables 1 and 2 . Those cases were retrieved spanning four years and included all needle biopsies performed at the Urology Department at our institution among 465 sequential biopsies (1.5\%). The mean age at diagnosis was 69.8 years. Metastatic NEC from any other site or direct extension from the bladder or gastrointestinal tract were excluded clinically in all cases. The serum PSA values at the time of initial diagnosis were available in 5 patients and ranged from 7.3 to 1449.0 $\mathrm{ng} / \mathrm{mL}$ (mean $461.1 \mathrm{ng} / \mathrm{mL}$, median $194.15 \mathrm{ng} / \mathrm{mL}$ ).

There were five small cell carcinomas and two tumors with morphology and mitotic count compatible with low-grade neuroendocrine carcinomas (carcinoid). There was no significant difference in tumor extent in biopsies between small cell carcinomas and carcinoid tumors. The numbers of cores obtained in the needle biopsies diagnosed with prostatic neuroendocrine tumors were six in two cases, eight cores in four cases and 12 cores in one case. NEC were associated with an acinar component in five of seven cases and the Gleason score of the acinar component was always $>6$ (Figures 1-3). One patient had a Gleason score of $3+4$, a second $4+4$, and a third 5+4 (Table-1). The five tumors with associated acinar adenocarcinomas in the biopsies involved a higher number of cores than the two tumors without an acinar component, but the difference was not significant. NEC tended to fragment in needle cores, and histologic crush artifact was also a common feature (Figure-4). Tumor necrosis was common and present at least focally in six of seven cases (Figure-5). Cytologically, tumor cells were small, with scant cytoplasm and open chromatin, with inconspicuous nucleoli (Figure-6). Immunohistochemical findings included positivity for neuroendocrine epitopes (chromogranin and synaptophysin) in all tumors with varying degrees of positivity. Chromogranin was stronger overall. The Ki-67 proliferative index varied from 40 to $90 \%$ in 


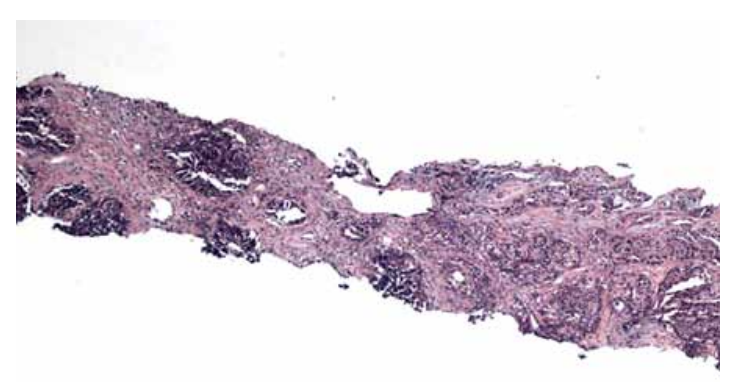

Figure 1 - Low power of small cell carcinoma (left) associated with Gleason 8 acinar adenocarcinoma (right). Hematoxilineosin, 40x.

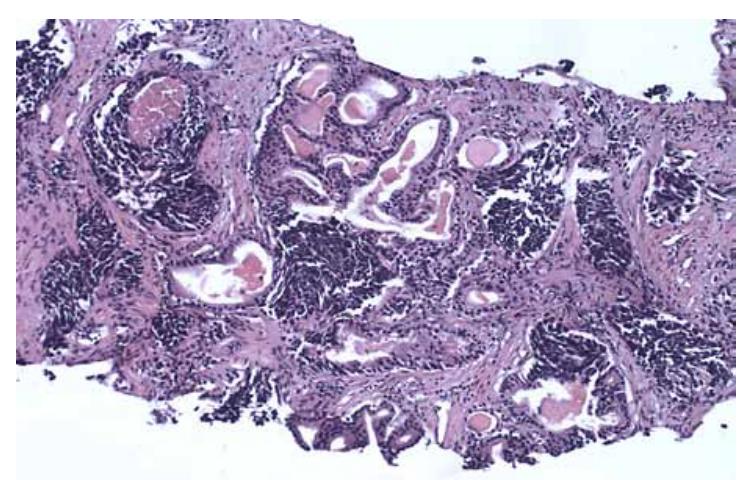

Figure 3 - Small cell carcinoma in close association with Gleason 4+3 acinar adenocarcinoma (same case as Figure-4). Hematoxilin-eosin, 100x.

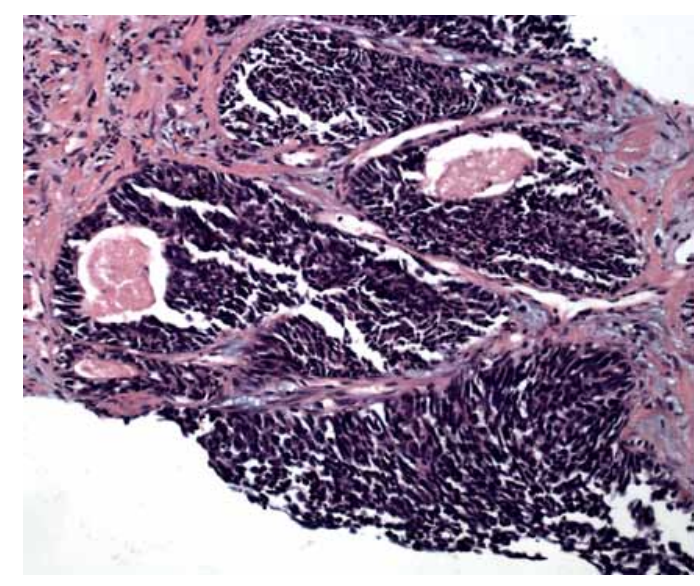

Figure 5 - Small cell carcinoma. Punctuate tumor necrosis is a common finding. Also note the desmoplastic stroma surrounding tumor nests. Hematoxilin-eosin, 200x.

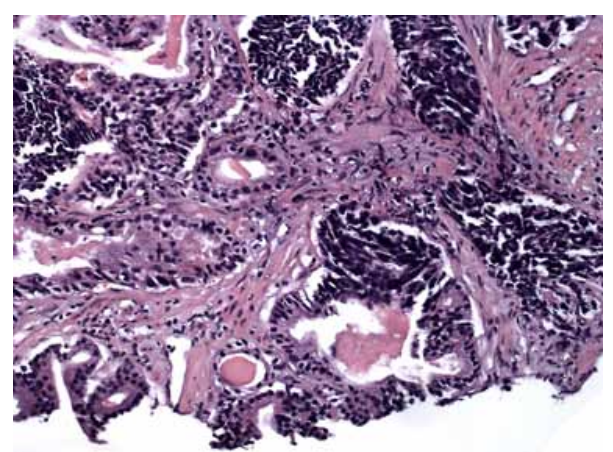

Figure 2 - Small cell carcinoma associated with acinar adenocarcinoma. On the upper right corner, small cell carcinoma predominates, whereas acinar Gleason pattern 3 can be seen in the left lower corner. Hematoxilin-eosin, 200x.

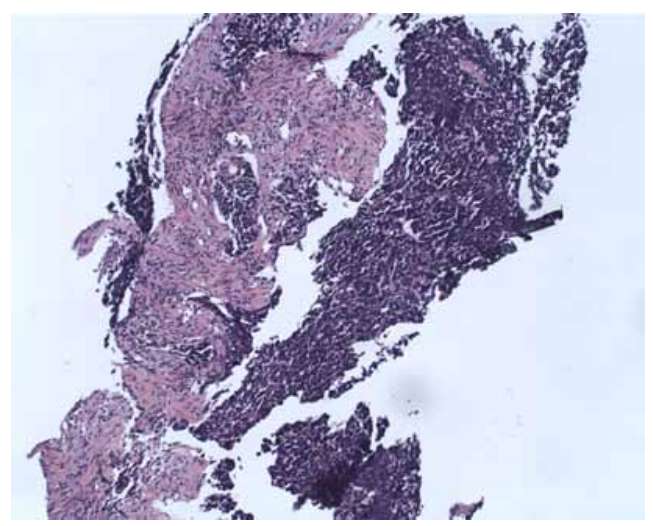

Figure 4 - Medium-power view of small cell carcinoma. The tumor tends to fragment on processing. Hematoxilin-eosin, 100x.

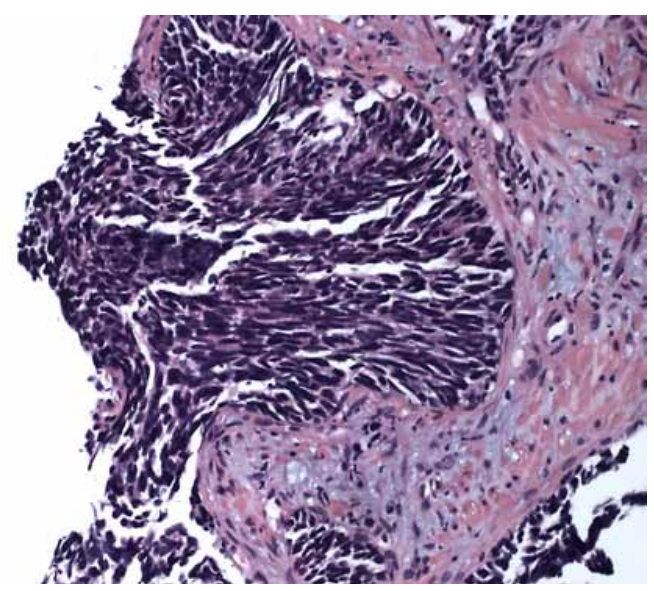

Figure 6 - Small cell cacinoma, cytologic findings. Small cell carcinoma. Nucleoli are inconspicuous and cytoplasm are scant. Hematoxilin-eosin, 200x. 


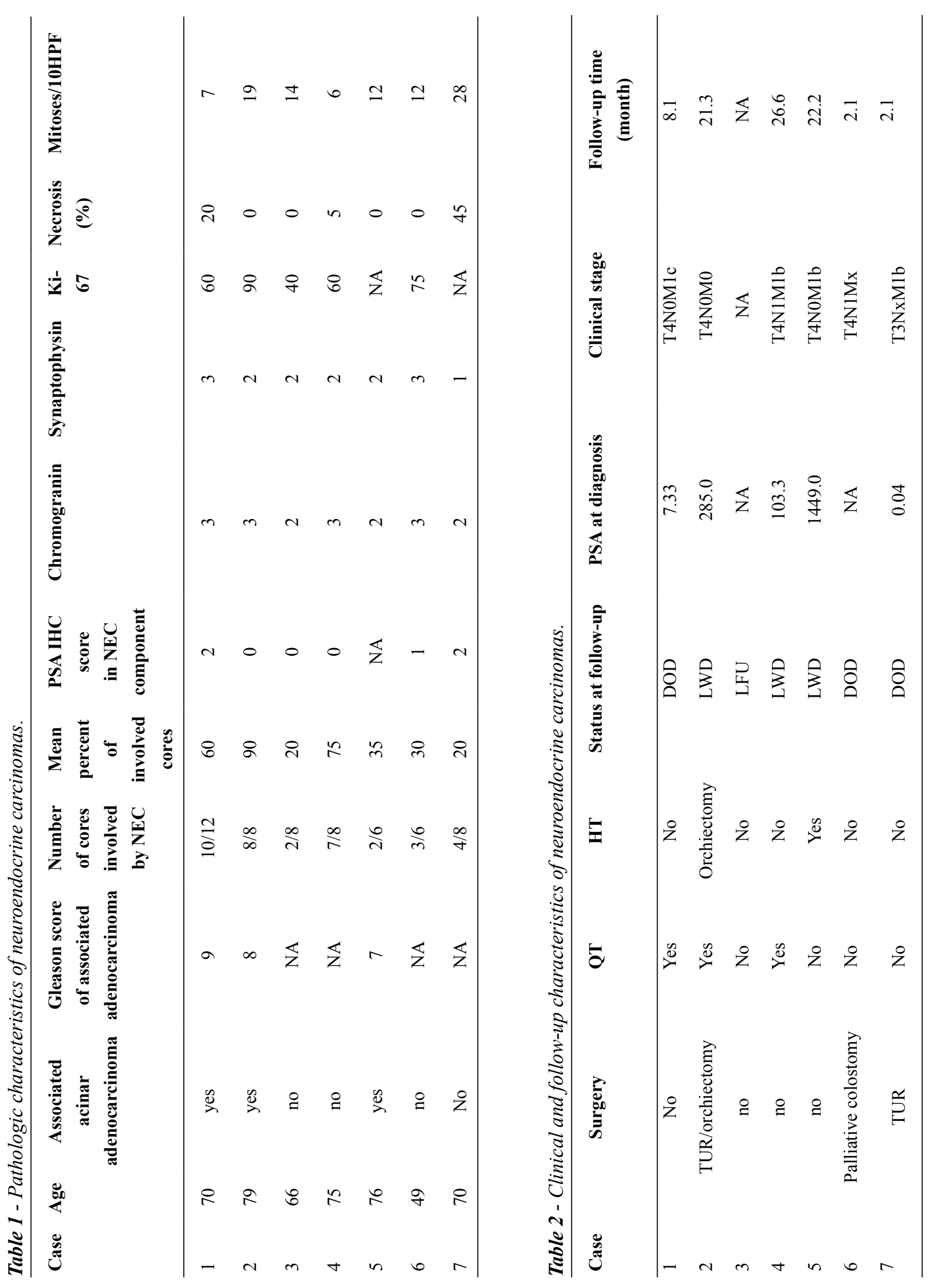


five available cases. PSA immunohistochemistry was only weakly positive in NEC in 2 cases (Figures 7-10).

During the same period, data on 458 conventional type acinar adenocarcinomas were reviewed and compared with the seven cases on this study. Of the 458 cases, there were 191 Gleason 6 , 131 Gleason 7, 72 Gleason 8, 33 Gleason 9 and 7 Gleason 10. The mean age at diagnosis correspondent to Gleason 6-10 were 69.1, 70.3, 74.2, 73.6 and $76.2(\mathrm{p}<0.001)$. Within the acinar tumors, high grade tumors (Gleason $>7$ ) also correlated with a high PSA at diagnosis $(\mathrm{p}<0.001)$, but not with prostate volume $(\mathrm{p}=0.3)$. The ratio of PSA/

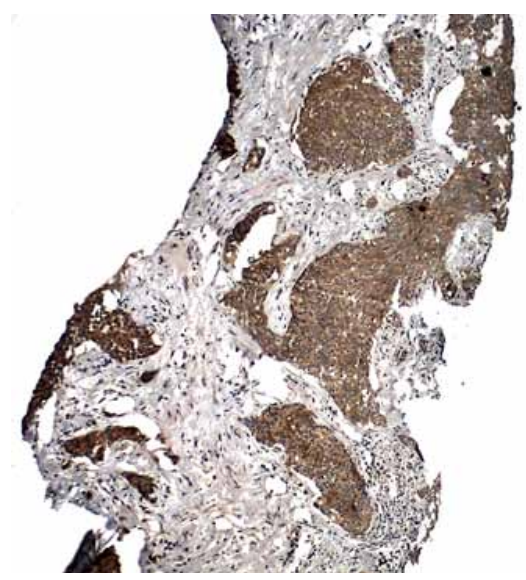

Figure 7 - High grade neuroendocrine carcinoma with strong positivity for chromogranin immunostain.

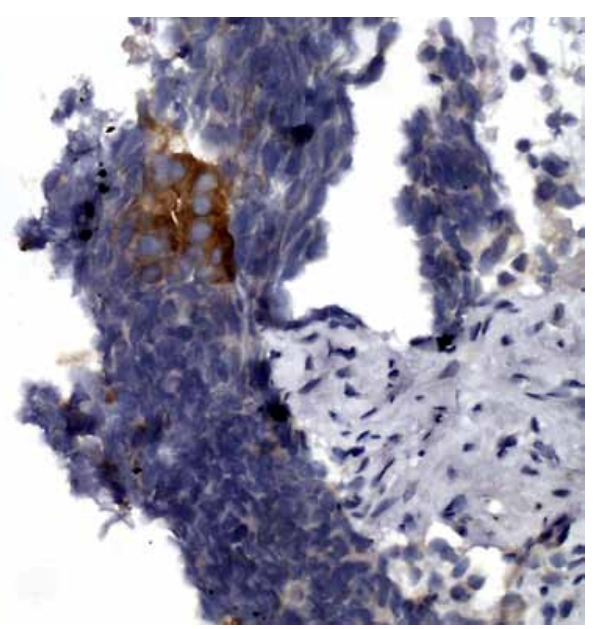

Figure 9 - Occasional neuroendocrine carcinoma tumor cells were positive for PSA immunostain. volume significantly correlated with a high Gleason score $(\mathrm{p}<0.05)$.

The number of cores involved in prostates with NEC was greater (65\% compared to $24 \%$ of acinar tumors, $\mathrm{p}<0.05)$. The mean PSA at diagnosis was 417.7 (range 5.7-1593, SD 218.3), compared to $100.5(\mathrm{p}=0.1)$ of acinar tumors (range 0.3-8545, SD 22.7). Prostates harboring NEC were bigger ( $\mathrm{p}<0.001$, mean volume $240 \mathrm{~mL}$ vs $53 \mathrm{~mL}$ of acinar tumors).

Follow-up was available in six patients. Treatment of our patients included chemotherapy and hormonal therapy, as well as palliative surgery. Most patients were diagnosed in an advanced

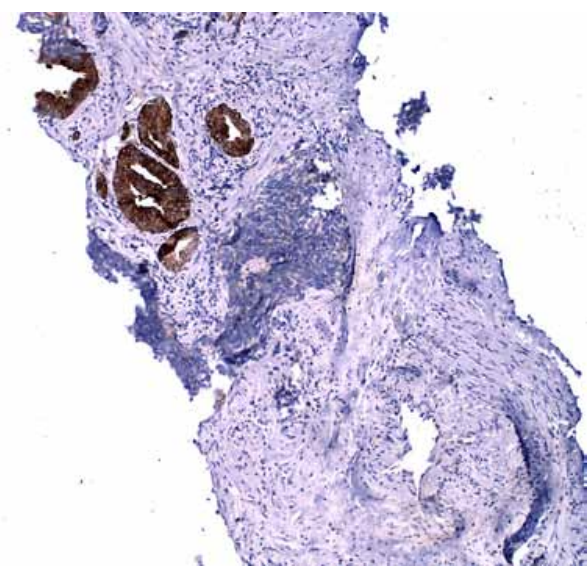

Figure 8 - PSA immunostain showing strong positivity in benign prostate glands, whereas the neuroendocrine tumor is faint to absent.

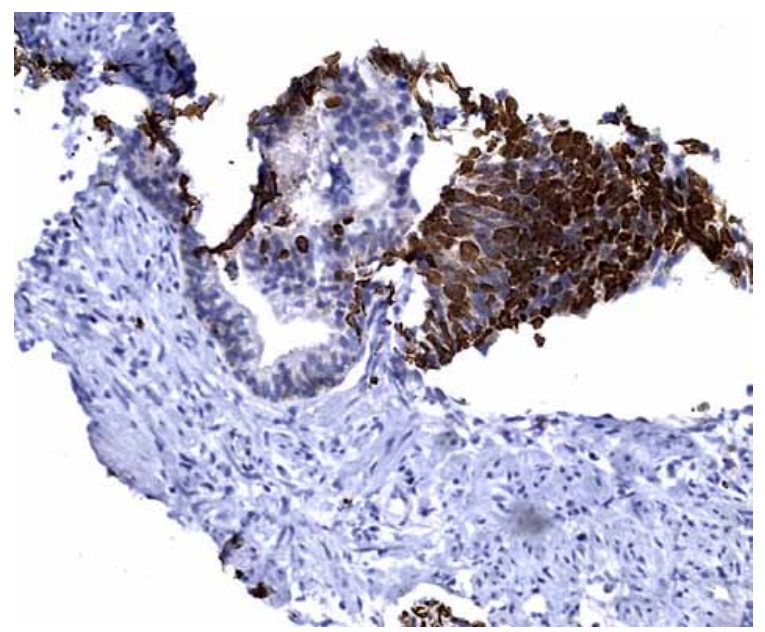

Figure 10 - Ki-67 showed a high proliferative index in most neuroendocrine tumors. 
stage, precluding the possibility of radical prostatectomy. Three patients underwent palliative surgery: one was treated with transurethral resection only for obstruction, a second with transurethral resection followed by surgical castration (orchiectomy) and a third with colostomy for intestinal obstruction by metastatic tumor. Three patients were treated with chemotherapy in association with etoposide phosphate (VP-16) and cisplatin (CDDP). In one of these patients, chemotherapy was suspended after one cycle due to obstructive renal failure, whereas in another patient the regimen was modified to taxol after the fourth cycle, but with no measurable response. Mean follow-up was 13.7 months, with a range of 2.1 months in two patients with distant metastatic disease at diagnosis (bone in one, and bone and liver in the second), to 26.6 months in a patients who is alive with disease at last follow-up (Table-2).

\section{DISCUSSION}

The morphologic features of NEC of the prostate are similar to those of other sites, including the common pulmonary small cell carcinomas (9). In prostate neuroendocrine carcinoma series, however, a common finding is the association with conventional acinar tumors, suggesting a common pathway of tumor differentiation, or a neuroendocrine transformation from the better-differentiated carcinoma to neuroendocrine tumor $(7,11)$. In the current study, only of the cases showed associated conventional type cancer; however, it is noteworthy that these were all diagnosed on needle biopsies, and one can not exclude another tumor components if the tumors were resected and examined throughout.

The diagnosis of high-grade neuroendocrine carcinomas in a needle biopsy may be challenging to the pathologists, especially because of the important clinical implication, which is exclusion from tentative surgical treatment with curative intent. None of the patients in the series were taken to radical prostatectomy, with two patients undergoing transurethral resection for obstructive disease palliative management. This data underscores the need for new therapeutic strategies to treat these tumors, which may include the use of protocols that have been effective against neuroendocrine carcinomas arising in other organ systems $(6,12-14)$.

In cases where the diagnosis of small cell carcinoma is difficult, either due to the limited materials available, or due to lack of clear neuroendocrine differentiation, where the main differential is always with high-grade Gleason 9 or 10 acinar prostate adenocarcinomas, and poorly differentiated urothelial tumors invading the prostate, immunohistochemistry can be helpful. The vast majority of these tumors express at least one neuroendocrine marker. Wang et al. reported a rate of $94 \%$, being CD56 the most sensitive (7). One caveat is that conventional adenocarcinomas, up to $100 \%$ in some studies, may focally express these same markers, reinforcing the need for careful morphologic evaluation by the pathologist $(5,15,16)$. More recent discoveries have suggested that prostatic specific membrane antigen (PSMA), CD44 and protein (P501S) may help with indentifying neuroendocrine expression in tumors $(7,17)$.

High-grade neuroendocrine carcinomas have been reported in association with obstructive symptoms in the setting of androgen-independent disease. In this scenario, serum PSA levels tend to be low to undetectable. In the current series, all cases were diagnosed de novo with a high mean PSA, indicating that those tumors or the associated acinar tumor are able to express high quantities of PSA. Interestingly, we have found no association of serum PSA levels and PSA detection on the tissue by immunohistochemistry (Tables 1 and 2).

\section{CONCLUSIONS}

Prostates harboring neuroendocrine carcinoma tend to be larger and involve a greater number of cores than acinar tumors. Association with conventional acinar tumors is common. Serum PSA levels vary greatly and its value at diagnosis does not seem to predict the presence of NE tumors in needle biopsy.

\section{CONFLICT OF INTEREST}

None declared. 


\section{REFERENCES}

1. Tanaka M, Suzuki Y, Takaoka K, Suzuki N, Murakami S, Matsuzaki O, et al.: Progression of prostate cancer to neuroendocrine cell tumor. Int J Urol. 2001; 8: 431-6; discussion 437.

2. Sarma DP, Weilbaecher TG: Small-cell carcinoma of prostate. Urology. 1989; 33: 332-5.

3. Oesterling JE, Hauzeur CG, Farrow GM: Small cell anaplastic carcinoma of the prostate: a clinical, pathological and immunohistological study of 27 patients. J Urol. 1992; 147: 804-7.

4. Manson AL, Terhune D, MacDonald G: Small cell carcinoma of prostate. Urology. 1989; 33: 78-9.

5. di Sant'Agnese PA, Cockett AT: Neuroendocrine differentiation in prostatic malignancy. Cancer. 1996; 78: 357-61.

6. di Sant'Agnese PA: Neuroendocrine differentiation in carcinoma of the prostate. Diagnostic, prognostic, and therapeutic implications. Cancer. 1992; 70(1 Suppl): 254-68.

7. Wang W, Epstein JI: Small cell carcinoma of the prostate. A morphologic and immunohistochemical study of 95 cases. Am J Surg Pathol. 2008; 32: 65-71.

8. Helpap B, Köllermann J, Oehler U: Neuroendocrine differentiation in prostatic carcinomas: histogenesis, biology, clinical relevance, and future therapeutical perspectives. Urol Int. 1999; 62: 133-8.

9. Brambilla E, Travis WD, Colby TV, Corrin B, Shimosato Y: The new World Health Organization classification of lung tumours. Eur Respir J. 2001; 18: 1059-68.

10. Shah RB, Mehra R, Chinnaiyan AM, Shen R, Ghosh $\mathrm{D}$, Zhou M, et al.: Androgen-independent prostate cancer is a heterogeneous group of diseases: lessons from a rapid autopsy program. Cancer Res. 2004; 64: 9209-16.

11. Evans AJ, Humphrey PA, Belani J, van der Kwast TH, Srigley JR: Large cell neuroendocrine carcinoma of prostate: a clinicopathologic summary of 7 cases of a rare manifestation of advanced prostate cancer. Am J Surg Pathol. 2006; 30: 684-93.
12. Stein ME, Bernstein Z, Abacioglu U, Sengoz M, Miller RC, Meirovitz A, et al.: Small cell (neuroendocrine) carcinoma of the prostate: etiology, diagnosis, prognosis, and therapeutic implications--a retrospective study of 30 patients from the rare cancer network. Am J Med Sci. 2008; 336: 478-88.

13. Segawa N, Inamoto T, Ibuki N, Mizutani Y, Azuma $\mathrm{H}$, Tsuji M, et al.: Neuroendocrine differentiation in adenocarcinoma of the prostate during hormonal treatment : a case report. Hinyokika Kiyo. 2010; 56: 49-54.

14. Köllermann J, Helpap B: Neuroendocrine differentiation and short-term neoadjuvant hormonal treatment of prostatic carcinoma with special regard to tumor regression. Eur Urol. 2001; 40: 313-7.

15. Ordóñez NG: Value of thyroid transcription factor-1 immunostaining in distinguishing small cell lung carcinomas from other small cell carcinomas. Am J Surg Pathol. 2000; 24: 1217-23.

16. Yao JL, Madeb R, Bourne P, Lei J, Yang X, Tickoo S, et al.: Small cell carcinoma of the prostate: an immunohistochemical study. Am J Surg Pathol. 2006; 30: 705-12.

17. Simon RA, di Sant'Agnese PA, Huang LS, Xu H, Yao JL, Yang Q, et al.: CD44 expression is a feature of prostatic small cell carcinoma and distinguishes it from its mimickers. Hum Pathol. 2009; 40: 252-8.

Submitted for publication:

January 12, 2011

Accepted after revision:

April 15, 2011

Correspondence address:

Dr. Marcos Venicio Lima

Hosp. do Câncer do Inst do Câncer do Ceará

Rua Papi Júnior, 1222

Fortaleza, CE, 60430-230, Brazil

E-mail:marcosvalima@hotmail.com 